\title{
Novel topical drug carriers as a tool for treatment of psoriasis: Progress and advances
}

\author{
Preeti K. Suresh*, Prameet Singh and Shailendra Saraf \\ University Institute of Pharmacy, Pt. Ravishankar Shukla University, Raipur, Chhattisgarh, 492010, India. \\ Accepted 10 January, 2013
}

\begin{abstract}
Psoriasis is a chronic inflammatory skin disease portrayed by erythematous, papulosquamous lesions. It is characterized by excessive growth and aberrant differentiation of keratatinocytes. The conventional topical treatments that have been used in the past, such as coal tar and dithranol, have low efficacy, poor aesthetic and cosmetic appeal, leading to poor patient compliance while systemic therapies such as methotrexate, cyclosporine and acitretin produce significant side effects. In recent years, several novel carriers like liposomes, nanostructured lipid carriers (NLC), etc. have been used in psoriasis, with promising results. Small and relatively narrow size distribution with novel carriers permits site specific delivery to the skin, with improved drug solubilization of hydrophobic drugs and bioavailability. This review highlights the recent advancements in the field of novel carriers for topical applications of antipsoriatic active moieties and bioactives.
\end{abstract}

Key words: Novel carriers, topical, antipsoriatic drugs, psoriasis, skin.

\section{INTRODUCTION}

Psoriasis is a psychosocially, and at times medically, debilitating disorder that affect 1 to $3 \%$ of the population world wide (Afifi et al., 2005). Psoriasis has a serious impact on health related quality of life, including physical and emotional well-being (Golant and Guttman-Yassky, 2011). It basically involves excessive growth and deviant differentiation of ketatinocytes (Lowes et al., 2007). Psoriasis pathogenesis is closely associated with disease-including $\mathrm{T}$ helper 1 (Th1) and $\mathrm{T}$ helper 17 (Th17) cells (Heidenreich et al., 2009). About $80 \%$ of patients with psoriasis vulgaris are treated topically (Peeters et al., 2005). The conventional topical medications in the management of psoriasis are fraught with several limitations, which the novel carriers are reported to circumvent with safe and long term use (Witman, 2001; Prohić et al., 2007). Novel carriers such as liposome, niosome, microemulsion, nanoemulsion, nanostructured lipid carrier and ethosomes have indeed brought us closer to the goal of safe and efficacious

\footnotetext{
*Corresponding author Email: preeti_venugopalan@yahoo.co.in, suresh.preeti@gmail.com. Tel: +91-98279-38427. Fax: +91771-2262832.
}

treatment of the disease (Katare et al., 2010).

\section{Pathophysiology of psoriasis}

Psoriasis is an immunologically mediated disease caused by activation of $\mathrm{T}$ lymphocytes in dermis (primarily CD4+ cells) and epidermis (predominantly CD8+ cells) (Nickoloff, 1999). The activation of T-cells is dependent upon its binding with the antigen presenting cells (APCs). This process is mediated through surface molecules used for adhesions, including leukocyte function associated antigen (LFA)- 1 and CD2 on the T cells and intercellular adhesion molecule (ICAM)-1, and LFA-3 on the APCs (Lowes et al., 2004).

The T-cell receptor (TCR) for the specific T-cell recognizes an antigen presented on the major histocompatibility complex (MHC I or II) by the APC. Activated $T$ cells proliferate and enter the circulation via trafficking through the interaction between LFA-1 and ICAM-1, and extravasate via diapedesis through the endothelium at site of inflammation in the skin. At this point, the T-cells propagate the immunologic process through the secretion of pro-inflammatory (type 1 or Th1) cytokines, which include interleukin (IL)-1, tumor necrosis 
Table 1. Classification of types of psoriasis and their features (Naldi and Gambini, 2007).

\begin{tabular}{|c|c|c|}
\hline $\mathbf{S} / \mathbf{N}$ & Type & Features \\
\hline 1 & Plaque & $\begin{array}{l}\text { Scalp lesions are well-marginated, scaly plaques. At times severe hyperkeratosis can accumulate, tightly } \\
\text { bound down by hair. Permanent hair loss is seldom seen, but may occur when excessive traction is applied } \\
\text { to the hair to scale. }\end{array}$ \\
\hline 2 & Pustular & $\begin{array}{l}\text { Primarily seen in adults. It may be localized to certain areas of the body, e.g. hands and feet. Can be } \\
\text { generalized, covering most of the body. It tends to go in a cycle as reddening of the skin followed by } \\
\text { formation of pustules and scaling. }\end{array}$ \\
\hline 3 & Guttate & $\begin{array}{l}\text { Appears as small, red, separate spots on the skin. Lesions usually appear on the trunk and limbs and } \\
\text { commonly number in the hundreds. Sometimes lesions form on the scalp, face and ears. They are not } \\
\text { usually as thick as the lesions that characterize plaque psoriasis. This form can precede or co-exist with } \\
\text { other forms of psoriasis, such as plaque. }\end{array}$ \\
\hline 4 & Flexural & $\begin{array}{l}\text { Differs from lesions elsewhere in that, although it is erythematous, it is not usually scaly. Exudation may be } \\
\text { prominent. }\end{array}$ \\
\hline 5 & Erythrodermic & $\begin{array}{l}\text { It is the least common type of psoriasis and may occur once or more during a lifetime in } 1 \text { to } 2 \text { percent of } \\
\text { people who develop psoriasis. It generally appears on people who have unstable plaque psoriasis. This } \\
\text { means the lesions are not clearly defined. Widespread, fiery redness and exfoliation of the skin characterize } \\
\text { this form. Severe itching and pain often accompanies it. }\end{array}$ \\
\hline
\end{tabular}

factor (TNF)- $\alpha$, and interferon (IFN)- $\mathrm{\gamma}$. These cytokines secretion results in the production of Th2 cytokine including IL-4, IL-10 and IL-11. Each cytokine down regulates the other's responses (Adorini and Trembleau, 1997). The final outcome of this bio-event is the formation of the psoriasis plaque through keratinocyte proliferation, an increase of activity and migration of other inflammatory cells and vascular changes (Hern et al., 2001).

Psoriasis has been considered basically as a disease of the keratinocyte, owing to its major clinical features of abnormal scaling and epidermal thickening. Clinically, the vascular nature of psoriasis is manifested by the erythematous plaques which exhibit pinprick bleeding on removal of scale (Auspitz sign), and is seen histologically with dilated tortuous capillaries, a prominent early feature of plaques. Histological studies have shown a fourfold increase in surface area of the superficial vascular plexus in psoriasis, and an increase in endothelial cell proliferation (Creamer, 1997). Angiogenic factors overexpressed by lesional keratinocytes include vascular endothelial growth factor (VEGF), IFN- $\gamma$, and IL-8 (IL8).

VEGF in particular, appears to play a central role in angiogenesis in psoriasis. This cytokine is also a potent mediator of inflammation and increases vascular permeability. Its synthesis by keratinocytes, activated T cells and endothelial cells, is induced by cytokines such as tumor growth factor- $\alpha$ (TGF- $\alpha$ ), IFN- $\gamma$, and TNF- $\alpha$. VEGF is over-expressed in psoriatic epidermis, and its receptors (KDR and Flt-1) are over-expressed on papillary microvessels. Transgenic mice over expressing VEGF in the epidermis were reported to develop a psoriasis-like condition (Xia et al., 2003). Serum concentrations of VEGF are increased in erythrodermic psoriasis (Creamer et al., 1996), and a correlation between plaques VEGF, psoriasis area and severity index (PASI) and serum VEGF values was also reported (Creamer et al., 2002). The hyperpermeability induced by VEGF has been proposed as the cause of microalbuminuria and pulmonary edema in patients with severe psoriasis.

The main factors responsible for psoriasis therefore include the angiogenic factors, over-expression of VEGF in the psoriatic epidermis, transfer in bone marrow transplants from affected individuals, reduced TNF concentrations and increased concentrations of natural killer (NK T) cells.

Psoriasis has been classified into several types as presented in Table 1. Table 2 lists the various drugs that have been used for psoriasis.

\section{Need for novel carriers}

Presently, it has become increasingly apparent that the development of new drug alone is not sufficient to ensure progress and success in drug therapy, since the main reasons for the failure of therapy remain poor drug solubility, insufficient drug concentration due to poor absorption, rapid metabolism and elimination, drug distribution to other tissues combined with high drug toxicity, and high fluctuation of plasma level due to unpredictable bioavailability after per oral administration, including the influence of food on plasma levels (Mehnert and Mader, 2012). A promising strategy to overcome 
Table 2. Various drugs for psoriasis with their mechanism of action and side effects.

\begin{tabular}{|c|c|c|c|}
\hline Compound & Mechanism of action & Side effect & Reference \\
\hline Coal tar & DNA suppression & Messiness, staining, odor, irritant, allergic, phototoxic responses and folliculitis & Walter et al. (1978) \\
\hline Psoralens & Suppresses DNA synthesis by cross-linking DNA strands & Long term risks include skin cancer and premature ageing of skin & Greaves et al. (1978) \\
\hline 5-Aminolevulinic acid & Antiproliferative effect & Photosensitivity and irritation & Ibbotson (2002) \\
\hline Anthralin & Antiproliferative effect & Irritation and staining of the skin, nails and clothing & Fuchs et al. (1990) \\
\hline Tazarotene & Antiproliferative and anti-inflammatory activities & Pruritis, erythema, burning and desquamation & Weinstein et al. (2003) \\
\hline Vitamin $\mathrm{D}$ analogues & Keratinocyte proliferation, enhance cellular differentiation & Irritation and hypercalcemia & Takahashi et al. (2003) \\
\hline Acitretin & Normalizes keratinocytes proliferation and differentiation & Mucocutaneous reactions, pruritus and hypertriglyceridemia & Rossi and Pellegrino (2009) \\
\hline Corticosteroids & Anti-inflammatory and immunosuppressant & Burning, skin atrophy, photosensitivity and irritation & Lebwohl et al. (2005) \\
\hline Methotrexate & Anti-inflammatory and immunosuppressant & Myelosuppression, hepatic fibrosis and pulmonary fibrosis & Leon et al. (2007) \\
\hline Cyclosporine & Immunosuppressant & The risk of infection, with long term use and non-melanoma skin cancer & Heydendael et al. (2003) \\
\hline Tacrolimus & Immunosuppressant & Burning, itching, skin cancer and lymphoma & Scheinfeld (2004) \\
\hline Pimecrolimus & Immunosuppressant & Burning, itching, skin cancer and lymphoma & Scheinfeld (2004) \\
\hline
\end{tabular}

these problems includes development of suitable drug carrier system to achieve controlled and localized delivery of the active drug according to the specific need of the therapy. The size of the carriers designed depends on the desired route of administration, and ranges from few nanometers (colloidal carriers) to the micrometer range (microparticles), and to several millimeters (implants). Implants and microparticles are too large for drug targeting and intravenous administration (Nanjwade and Patel, 2011). Therefore, colloidal carriers have attracted increasing attention during the recent years. Investigated systems include nanoparticles, nanoemulsions, microemulsions, liposomes, ethosomes, nanosuspensions, micelles and soluble polymer-drug conjugates.

\section{Topical drug carriers and challenges in dermal delivery}

During the last decades, inorganic and colloidal particles such as nanocapsules, nanospheres, nanostructured lipid carrier, etc. have been explored for dermal/transdermal drug delivery. The successful implementation of these systems for drug delivery completely depends on their ability to penetrate through several anatomical barriers, sustained release of their content and their stability in the nanometer size. Different colloidal drug delivery systems (including liposomes, dendrimers and polymeric nanoparticles, in addition to solid lipid nanoparticles), were developed to overcome physicochemical limitations of potential therapeutic compounds such as poor solubility, low permeability, short half-life, high molecular weight, side effects and systemic toxicity (Almeida and Souto, 2007)

Stratum corneum is the main barrier in the percutaneous absorption of topically applied drugs. Small and relatively narrow size distribution with novel carrier permit site specific delivery to the skin with improved drug solubilization of hydrophobic drugs and better bioavailability. The problem of lipid imbalance can be resolved by delivering the unsaturated fatty acids like linoleic acid to restore the normal skin conditions by lipid carriers (Morganti et al., 2001). This provides lipid enriched hydrating conditions to help retain the drug molecules within the dermal layers at or near to the site of action.

\section{NOVEL CARRIERS USED IN PSORIASIS}

In recent years, attempts have been made for successful delivery of antipsoriatic agents by employing novel carriers (Table 3).

\section{Liposome}

Liposomes are microscopic structures consisting of one or more concentric spheres of lipid bilayers enclosing aqueous compartments. As drug carrier 
systems for topical treatment, liposomes are reported to be superior over conventional topical preparations. Phospholipids, being the major component of liposomal systems, are easily integrated with the skin lipids and maintain the desired hydration conditions to improve drug penetration and localization in the skin layers (Moghimi and Patel, 1993; Cevc et al., 1996; Schmid and Korting, 1996).

The use of liposomes as drug carriers seems to be promising, exhibiting therapeutic prospects with a reduction of side effects. Liposomal encapsulation of retenoids, for example vitamin A acid or tretinoin reduced the local irritation (Trapasso et al., 2009). Reports suggest that liposomal encapsulation of tacrolimus in topical formulation enhanced its penetration through skin. These characteristics may allow liposomal tacrolimus to be effective against psoriasis without the need of occlusive dressings. If liposomal tacrolimus penetrate into the blood stream, reports with other models suggest that it will be less toxic than free tacrolimus. This will be more important to patients who require higher concentrations of ointment to large areas of skin (Patel et al., 2010). Bhatia et al. (2004) developed and characterized tamoxifen loaded liposome for topical therapy and reported improved skin permeation of Tamoxifen (TAM) and retention in the skin.

Calcipotriol, a vitamin $\mathrm{D}$ analogue was successfully delivered in lipopolymer poly(ethylene glycol)distearoylphosphoethanolamine (PEG-DSPE) liposomes with a significant increase in drug deposition into the stratum corneum (Knudsen et al., 2012). The size of the liposomes affected the penetration of calcipotriol into the stratum corneum, as smaller unilamellar vesicles enhanced drug penetration as compared to large multilamellar vesicles. This also indicated that the liposomes to some extent migrate as intact vesicles into the stratum corneum. Srisuk et al. (2012) compared the physicochemical characteristics and in vitro permeability of methotrexate entrapped deformable liposomes prepared from phosphatidylcholine (PC) and oleic acid, with conventional liposomes prepared from PC and cholesterol $(\mathrm{CH})$. The deformable liposomes displayed enhanced permeability and this was attributed to the elastic characteristics of the oleic acid containing liposomes, as well as skin penetration enhancer effect of oleic acid.

Nagle et al. (2011) investigated the potential benefits of combining menthol with methotrexate in a vesicular gel base for improving the drug penetration, its dermal availability and patient acceptability. Antipsoriatic efficacy of the formulations tested in vivo, using the rat tail model, indicated that the vesicular gel containing menthol led to maximum drug retention in the skin. The in vivo studies also ascertained the effectiveness of the formulation in inducing a normal pattern of differentiation in the rat tail skin that initially showed parakeratosis, which is also characteristic of psoriatic epidermis.
Li et al. (2010) reported an ultra deformable cationic liposome for transdermally delivering plasmid DNA to mouse skin. The antipsoriatic efficacy in the K14-VEGF transgenic mouse model by transdermal delivery of murine IL-4 was observed. Plasmid DNA was transdermally delivered to vicinal sites of epidermis and hair follicles. Plasmid DNA expression was detected in ear skin.

\section{Niosome}

The low cost, greater stability and ease of storage of nonionic surfactants led to the exploitation of these compounds as alternative to phospholipids, the main constituent of liposomes (Uchegbu and Vyas, 1998). Niosomes are microscopic lamellar structures formed on admixture of a non ionic surfactant, cholesterol and a charge inducing agent, with subsequent hydration in aqueous media (Uchegbu and Florence, 1995; D'Souza et al., 1997). Niosomes comprises of an architecture consisting of both hydrophobic and hydrophilic moieties, and as a result can accommodate drug molecules of varying solubilities (Namdeo and Jain, 1996). Niosomes have been evaluated in many pharmaceutical applications and were reported to reduce systemic toxicity by drug encapsulation and minimize clearance of such agents from the body by slow drug release. Agarwal et al. (2001) developed dithranol entrapped in liposomal and niosomal vesicles $(0.5 \%)$, and found both of them superior to conventional formulation, while liposomes showed better results than niosomes employing mice skin. Marianecci et al. (2012) investigated the niosomes made up of surfactants (Tween 85 and Span 20) and cholesterol for the delivery of ammonium glycyrrhizinate (AG), useful for the treatment of various inflammatory based diseases. The in vitro/vivo efficacy of the ammoniu glycyrrhizinate/niosomes was studied in murine and human models of inflammation. The AG-loaded non-ionic surfactant vesicles showed no toxicity, good skin tolerability and were able to improve the drug antiinflammatory activity in mice. Furthermore, an improvement of the anti-inflammatory activity of the niosome delivered drug was observed on chemically induced skin erythema in humans.

Niosomal methotrexate in chitosan gel was tested for irritation and sensitization on healthy human volunteers, followed by its assessment through double-blind placebocontrolled study on psoriasis patients (Lakshmi et al., 2007). The human repeated insult patch test did not produce any significant irritation or sensitization on healthy human volunteers. The placebo and marketed gels were compared with niosomal methotrexate gel. At week 12, with niosomal methotrexate gel, there was reduction in total score from $6.2378 \pm 1.4857$ to $2.0023 \pm$ 0.1371 . These results suggested better efficacy of niosomal methotrexate gel as compared to placebo and marketed methotrexate gel. 
Table 3. Previous work on novel carriers for psoriasis.

\begin{tabular}{|c|c|c|c|c|}
\hline Drug & Limitations of conventional form & Novel carrier & Effect observed & Reference \\
\hline \multirow[t]{2}{*}{ Tacrolimus } & \multirow[t]{2}{*}{ Normal skin transport } & Liposome & $\begin{array}{l}\text { Facilitated entry into the tough barrier consisting of stratum corneum. Better } \\
\text { solubility of drug }\end{array}$ & Patel et al. (2010) \\
\hline & & Nanolipid carrier & Provide good occlusion property and solubility & Pople and Singh (2011) \\
\hline Coal Tar & Skin irritation and staining & Phospholipid lipid carrier & Reduce irritation and better solubility & Bhatia et al. (2008) \\
\hline \multirow{3}{*}{ Anthralin } & \multirow{3}{*}{ Irritation and staining } & Liposome & Increase the stability of drug. Increases the penetration of drug through skin & Saraswat et al. (2007) \\
\hline & & Niosome & Enhance stability of drug & Agrawal et al. (2001) \\
\hline & & Liposome & Increases drug uptake and target deeper skin layer & Mahrle et al. (1991) \\
\hline Vitamin D analogues & Local irritation & Nanostructured lipid carrier & Enhanced skin permeation and negligible skin irritation & Lin et al. (2010) \\
\hline \multirow{2}{*}{ Corticosteroids } & \multirow{2}{*}{ Skin irritation } & Nanocapsule suspension & Greater stability of drug & Fontana et al. (2010) \\
\hline & & PEG-NLCs & High entrapment efficiency and improved drug stability & Doktorovova et al. (2010) \\
\hline \multirow{4}{*}{ 5-aminolevulinic acid } & \multirow{4}{*}{ Poor skin penetration } & Liposome & Enhance drug uptake & Casas and Batlle (2006) \\
\hline & & Liposome & Compatible with skin lipid & Venosa et al. (2008) \\
\hline & & Ethosome & Better skin permeation and targeting deeper skin layer & Fang et al. (2009) \\
\hline & & Nanoemulsion & optimize topical drug permeation negligible skin disruption and acceptable safety & Zhang et al. (2011) \\
\hline \multirow{2}{*}{ Temoporfin } & \multirow{2}{*}{ Low aq. solubility } & Liposome & Higher rate of drug transfer across skin & Dragicevic-Curic et al. (2009a) \\
\hline & & Invasome & Enhanced drug uptake & Dragicevic-Curic et al. (2009b) \\
\hline Psoralens & Low penetration & Microemulsion & Enhanced drug uptake and provide photostability & Baroli et al. (2000) \\
\hline \multirow{5}{*}{ Methotrexate } & \multirow{5}{*}{ Low penetration } & Liposome hydrogel & Enhance drug uptake & Ali et al. (2008) \\
\hline & & Deformable liposome & Better skin permeation and targeting deeper skin layer & Trotta et al. (2004) \\
\hline & & Niosome & Enhance stability of drug & Lakshmi et al. (2007) \\
\hline & & Microemulsion & Enhanced drug uptake and provide photostability & Zhi and Jin (2011) \\
\hline & & Nanogel & Greater stability of drug & Ali et al. (2008) \\
\hline \multirow{2}{*}{ Cyclosporine } & \multirow{2}{*}{ Limited cutaneous permeation } & Microemulsion & Increase the stability of drug & Umezawa and Ozawa (2007) \\
\hline & & PLGA-nanoparticle & Rapid dissolution of drug and good stability & Jain et al. (2011) \\
\hline Acitretin & Scaling, erythema, burning, stinging & Nanostructured lipid carrier & No major systemic side effects, the main topical side effect was irritation & Agrawal et al. (2010) \\
\hline
\end{tabular}

\section{Microemulsion}

Microemulsions are thermodynamically stable isotropic systems in which two immiscible liquids (water and oil) are mixed to form a single phase by means of an appropriate surfactant or its mixture. The short to medium chain alcohols are generally considered as co-surfactants in the microemulsion system. The presence of surfactant and co-surfactant in the system makes the interfacial tension very low. Therefore, microemulsions form spontaneously, with an average droplet diameter of 10 to $140 \mathrm{~nm}$. Microemulsions have the ability to deliver larger amounts of water and topically applied agents into the skin than water alone or other traditional vehicles such as lotions or creams because they act as a better reservoir for a poorly soluble drug through their solubilization capacity. Although microemulsion can be used to deliver drugs via several routes, the system has been extensively 
studied as a vehicle for topical and transdermal administration (Peltola et al., 2003; Sintov and Botner, 2006; Kreilgaard, 2002).

In topical and transdermal formulations, microemulsions have increased the cutaneous absorption of both lipophilic and hydrophilic APIs when compared to conventional vehicles (emulsions, pure oils, aqueous solutions, etc). Due to their special features, microemulsions offer several advantages for pharmaceutical use, such as ease of preparation, longterm stability, high solubilization capacity for hydrophilic and lipophilic drugs, and improved drug delivery (Karasulu, 2008; Heusehkel et al., 2008).

Zhi and Jin (2011) prepared methotrexate loaded microemulsion and evaluated microemulsion quality and percutaneous penetration through the skin. Umezawa and Ozawa (2007) reported that cyclosporine microemulsion increased the percutaneous absorption in skin of patients with psoriasis. Baroli et al. (2000) reported that microemulsion for topical delivery of 8methoxsalen increased the photostability and enhanced the percutaneous absorption of drug. Alvarez-Figueroa and Blanco-Mendez (2001) investigated the effectiveness of transdermal administration of methotrexate (MTX) by iontophoretic delivery from two types of hydrogel and passive delivery from two types of microemulsion by in vitro assays. Both iontophoretic delivery of MTX from hydrogels and passive delivery from microemulsions were more effective than passive delivery from aqueous solutions of the drug. In the passive delivery assays, both water/oil (w/o) and oil/water (o/w) microemulsions were used, and the effectiveness of delivery from o/w systems was higher. At the end of all assays, significant amounts of MTX were detected in the skin.

Raza et al. (2011) developed and characterised a novel dithranol-containing phospholipid microemulsion for enhanced skin permeation and retention. The microemulsion systems composed of isopropyl myristate and Tween 80 , with mean particle diameter of $72.8 \mathrm{~nm}$ showing maximum skin permeation $(82.23 \%)$, skin permeation flux $\left(0.281 \mathrm{mg} / \mathrm{cm}^{2} / \mathrm{h}\right)$ along with skin retention $(8.31 \%)$ with regard to systems containing tocopherol acetate and Tween 20.

\section{Nanoemulsion and nanoemulsomes}

Nanoemulsions are obtained when the dimension of an emulsion globule reaches approximately 20 to $500 \mathrm{~nm}$. The small droplet size can resist the physical destabilization caused by gravitational separation, flocculation and/or coalescence. It also avoids the creaming process because the droplet's Brownian motion is enough to overcome the gravitational separation force. The size and polydispersity of nanoemulsions can affect properties such as particle stability, rheology, appearance, color, texture and shelf life. They are not formed spontaneously, and their properties depend on the thermodynamic conditions and preparation methods. Nano/submicron emulsions are well accepted for their ability to increase skin permeation, prolonged action on the skin, and protection of the drug from instability (Solans et al., 2005; Weyenberg et al., 2007).Zhang et al. (2011) formulated 5-aminolevulinic acid (ALA) and methyl 5-aminolevulinic acid (mALA) at equimolar concentrations in $\mathrm{o} / \mathrm{w}$ and $\mathrm{w} / \mathrm{o}$ nanoemulsions for skin delivery and showed that apart from drug, the emulsion type and the oil phase are all important considerations when attempting to optimize topical drug permeation. The o/w soybean oil dispersions were most promising because of their ability to exert the highest in vitro ALA flux. The uniformity of drug flux by the emulsions was improved when compared to the aqueous control. The addition of a-terpineol, a penetration enhancer, as a part of the oil phase did not further increase drug permeation via the skin.Khandavilli and Panchagnula (2007) formulated a nanoemulsion (NE) to achieve penetration of paclitaxel into deeper skin layers while minimizing the systemic escape. Upon dermal application, the drug was predominantly localized in deeper skin layers, with minimal systemic escape. Bernardi et al. (2011) formulated rice bran oil nanoemulsions and evaluated it for irritation potential and moisturising activity on volunteers with normal and diseased skin types.

Optimized dithranol-loaded emulsomes were found to substantially enhance the antipsoriatic activity on a mouse-tail model with regard to marketed product (Raza et al., 2012). Also, the selected composition enhanced drug permeation and marked skin retention. The formulation was found to be quite non-irritant, stable and biocompatible in comparison to the marketed product.

\section{Nanostructured lipid carrier}

Nanostructured lipid carriers (NLCs) are the latest generation of Solid lipid nanoparticles (SLNs) possessing improved properties of drug loading, modulation of the release profile, and stable drug incorporation during storage. NLCs are produced by mixing solid lipids with spatially incompatible lipids leading to a lipid matrix with a special structure. A blend of a liquid and solid lipid creates a less perfect crystalline structure with many imperfections, providing more space for drug accommodation. Examples of solid lipids include triglycerides (tristearine, tripalmitine, trimyristine), fatty acids (stearic acid, palmitic acid), waxes (carnauba, cetyl palmitate), whereas liquid lipids include medium chain triglycerides, oleic acid, isopropyl myristate. Their suitability for dermatological applications was also confirmed by successful formulations of drugs for treatment of skin diseases. NLC systems are a promising carrier for the topical delivery of antipsoriatic drugs as revealed by enhanced skin permeation, negligible skin irritation, and the compatibility of the drugs (Muller et al., 2007). 
Lin et al. (2010) combined calcipotriol and methotrexate in nanostructured lipid carriers for topical delivery, and reported efficient delivery of the drugs. Nam et al. (2007) investigated tacrolimus loaded nanostructured lipid carriers for topical delivery. The penetration rate of these NLCs through the skin of a hairless mouse was greater than a commercial dermal ointment containing tacrolimus. Fluticasone propionate in novel polyethylene glycol (PEG)-containing nanostructured lipid carriers in the presence of PEG in NLC composition improved the physicochemical stability of the developed NLC (Doktorovova et al., 2010).

\section{Ethosome}

Ethosomes are noninvasive delivery carriers that enable drugs to reach the deep skin layers and/or the systemic circulation. These are soft, malleable vesicles tailored for enhanced delivery of active agents. They are composed mainly of phospholipids, (phosphatidylcholine, phosphatidylserine, phosphatidic acid), high concentration of ethanol and water. It is reported that ethosomes penetrate the skin and allow enhanced delivery of various compounds to the deep strata of the skin or to the systemic circulation (Touitou, 2002).

The high concentration of ethanol in ethosomes causes disturbance of skin lipid bilayer organization, hence when incorporated into a vesicle membrane, it enhances the vesicle's ability to penetrate the stratum corneum. Also, because of their high ethanol concentration, the lipid membrane is packed less tightly than conventional vesicles but has equivalent stability, allowing a more malleable structure and improves drug distribution ability in stratum corneum lipids (Merdan et al., 1998). Trotta et al. (2004) prepared deformable liposomes for dermal administration of methotrexate, and these provided maximum dose administration through the skin that may be of value for topical administration of methotrexate in the treatment of psoriasis.

\section{Novasome}

Novasomes are the modified forms of liposomes or a variation of niosomes prepared from the mixture of monoester of polyoxyethylene fatty acids, cholesterol and free fatty acids at 74/22/4 ratio and are 0.1 to $1.0 \mu \mathrm{m}$ in diameter.

They consist of two to seven layered shells that surround an unstructured space occupied by a large amorphous core of hydrophilic or any water-soluble molecules that have been mixed with the hydrophobic materials (Singh et al., 2011). An agonist of parathyroid hormone related peptide receptor, PTH (1-34), was formulated in Novasome A cream (Holick et al., 2003). Novasome A cream enhanced the percutaneous absorption of PTH (1-34) in human skin in comparison with formulations in propylene glycol or normal saline. Psoriatic lesions treated with PTH (1-34) showed marked improvement in scaling, erythema and induration.

\section{Solid lipid nanoparticle}

During the last two decades, numerous reports have described various SLN formulations that may find applications in drug delivery. The SLN structure is composed of solid core, which may contain triglycerides, glyceride mixtures, or waxes that are solid at both room temperature and human body temperature. SLNs are interesting lipid based drug delivery carriers for a number of reasons, including their nanoscale particle size and their biocompatible and biodegradable components. Urban-Morlan et al. (2010) prepared and characterized solid lipid nanoparticle containing cyclosporine A (CyA) by emulsification-diffusion method. Gambhire et al. (2011) reported the preparation and optimization of dithranol loaded solid lipid nanoparticles. The results of the ex vivo penetration studies demonstrated about twofold increase in localization of delayed type IV hypersensitivity (DTH) in skin with SLN entrapped ointment compared to plain DTH.

\section{HERBAL DRUG USED FOR PSORIASIS}

World Health Organization (WHO) notes that $74 \%$ of the plant derived medicines are used in modern medicine in a way that their modern application directly correlates with their traditional use as herbal medicines by native cultures (Kumar et al., 2003). WHO estimated that 65 to $80 \%$ of the world's population uses traditional medicines as their primary form of health care, and about $85 \%$ of traditional medicines involve the use of herbal preparations.

Various bioactives and phytoconstituents have been delivered using novel carriers in psoriasis as displayed in Table 4.

\section{CONCLUSION}

Despite the significant advances that have been made in understanding the pathomechanisms of psoriasis and in identifying effective treatment approaches, the search for optimum treatment strategies for psoriasis still remains a major challenge.

Numerous topical treatment modalities are available for psoriasis, but there is a dearth of uniformly acceptable approach for moderate-to-severe psoriasis. A variety of lipid based novel drug delivery systems like lipid nanoparticles, microemulsion, nanoemulsions, nanoemulsomes, 
Table 4. Bioactives delivered through novel carriers for psoriasis.

\begin{tabular}{|c|c|c|c|}
\hline Bioactives & Novel carrier & Effect observed & Reference \\
\hline Psoralea carylifolia (babchi oil) & Microemulsion gel & Psoralea corylifolia seed oil was effective with improved stability & Kumar and Parmar (2003) \\
\hline Curcuminoids & Nanoparticles & Enhanced skin uptake and better retention & Mulik et al. (2009) \\
\hline Glycyrrhetic acid & Nanoemulsion & Increased the stability of formulation and better transdermal effect & Puglia et al. (2010) \\
\hline Tea tree oil & Microemulsion & Potential in enhanced drug uptake & Khokhra and Diwan (2011) \\
\hline Cholchicine & Liposome & Sustained delivery & Singh et al. (2009) \\
\hline 3,5-dihydroxy-4-isopropylstilbene & Nanoemulsion & Dramatically improved the DHPS transdermal effect, and the osmotic quantity of DHPS from the nanoemulsion formulations & Zhang et al., 2011 \\
\hline
\end{tabular}

liposomes, niosomes and nanostructured lipid carriers clearly present a tool to overcome the many challenges associated with topical antipsoriatic drug therapy.

These novel carriers have been widely studied and have demonstrated an increment in the rate and extent of drug delivery and optimal therapeutic outcomes. Thus, there is need for further investigations with antipsoriatic drugs to establish the clinical utility and industrial scale-up of techniques for manufacturing these potential novel carriers.

\section{REFERENCES}

Adorini L, Trembleau S (1997). Immune deviation towards Th2 inhibits Th1-mediated autoimmune diabetes. Biochem. Soc. Trans. 25:625-629.

Afifi T, Gannes GD, Huang C, Zhou Y (2005). Topical therapies for Psoriasis: Evidence-based review: Can. Fam. Physician 51:519-525.

Agrawal R, Katare OP, Vyas SP (2001). Preparation and in vitro evaluation of liposomal/ niosomal delivery system for antipsoriatic drug dithranol. Int. J. Pharm. 228:43-52.

Agrawal Y, Petkar KC, KK Sawant (2010). Development, evaluation and clinical studies of acitretin loaded nanostructured lipid carriers for topical treatment of psoriasis. Int. J. Pharm. 401:93-102.

Ali FM, Salah M, Rafea M, Saleh N (2008). Liposoma methotrexate hydrogel for treatment of localized psoriasis:
Preparation, characterization and laser targeting. Med. Sci. Monit. 14:PI66-74.

Ali J, Akhtar N, Sultana Y, Baboota S, Ahuja A (2008). Antipsoriatic microemulsion gel formulations for topical drug delivery of babchi oil (Psoralea corylifolia). Methods Find. Exp. Clin. Pharmacol. 30:277-285

Almeida AJ, Souto E (2007). Solid lipid nanoparticles as a drug delivery system for peptides and proteins. Adv. Drug Del. Rev. 59:478-490.

Alvarez-Figueroa MJ, Blanco-Mendez J (2001) Transdermal delivery of methotrexate: Iontophoretic delivery from hydrogels and passive delivery from microemulsions. Int. $\mathrm{J}$. Pharm. 215: 57-65

Baroli B, López-Quintela MA, Delgado-Charro MB, Fadda AM Blanco- Méndez J (2000). Microemulsion for topical delivery of 8-methoxsalen. J. Control Rel. 69: 209-218.

Bernardi DS, Pereira TA, Maciel NR, Bortoloto J, Viera GS, Oliveira GC, Rocha-Filho PA (2011). Formation and stability of oil-in-water nanoemulsions containing rice bran oil: In vitro and in vivo assessments. J. Nanobiotechnol. 9:44.

Bhatia A, Kumar R, Katare OP (2004). Tamoxifen in topica liposomes: Development, characterization and in vitro evaluation. J. Pharm. Pharm. Sci. 7:252.

Bhatia A, Mangat P, Jain B, Singh B, Katare OP (2008). Washability and fabric-staining properties of a novel phospholipid-structured coal tar formulation. J. Dermatol. Treat. 19:105-110

Casas A, Batlle A (2006). Aminolevulinic acid derivatives and liposome delivery as strategies for improving 5 aminolevulinic acid-mediated photodynamic therapy. Curr. Med. Chem. 13:1157-1168.

Cevc G (1996). Transferosomes, liposomes and other lipid suspensions on the skin: permeation enhancement, vesicle penetration, and transdermal drug delivery. Crit. Rev. Ther. Drug Carrier Syst. 13:257-388.
Creamer D, Allen M, Jaggar R, Stevens R, Bicknell R, Barker J (2002). Mediation of systemic vascular hyperpermeability in severe psoriasis by circulating vascular endothelial growth factor. Arch. Dermatol. 138:791-796.

Creamer D, Allen MH, Groves RW, Barker JN (1996) Circulating vascular permeability factor/vascular endothelia growth factor in erythroderma. Lancet. 348:1101.

Creamer D, Allen MH, Sousa A, Postan R, Barker JN (1997). Localisation of endothelial proliferation and microvascular expansion in active plaque psoriasis. Br. J. Dermatol. 136:859-865.

Venosa G, Hermida L, Batlle A, Fukuda H, Defain MV, Mamone L, Rodriguez L, Casas A (2008). Characterization of liposomes containing aminolevulinic acid and derived esters. J. Photochem. Photobiol. B. Biol. 92:1-9.

Doktorovova S, Araujo J, Garcia ML, Rakovsky E, Souto EB (2010). Formulating fluticasone propionate in novel PEGcontaining nanostructured lipid carriers. Coll. Surf. B: Biointerf. 75:538-542.

Dragicevic-Curic N, Scheglmann D, Albrecht V, Fahr A (2009a). Development of liposomes containing ethanol for skin delivery of temoporfin: characterization and in vitro penetration studies. Coll. Surf. B. Biointerf. 74:114-122.

Dragicevic-Curic N, Scheglmann D, Albrecht V, Fahr A (2009b). Development of different temoporfin-loaded invasomes-novel nanocarriers of temoporfin: characterization, stability and in vitro skin penetration studies. Coll. Surf. B. Biointerf. 70:198-206.

D'Souza SA, Ray J, Pandey S, Udupa N (1997). Absorption of ciprofloxacin and norfloxacin when administered as niosomes-encapsulated inclusion complexes. J. Pharm. Pharmacol. 49:145-149.

Fang YP, Huang YB, Wu PC, Tsai YH (2009). Topical delivery of 5- aminolevulinic acid-encapsulated ethosomes in a hyperproliferative skin animal model using the CLSM 
technique to evaluate the penetration behavior. Eur. J. Pharm. Biopharm.73:391-398.

Fontana MC, Bastos MO, Beck RC (2010). Development and validation of a fast RP HPLC method for the determination of clobetasol propionate in topical nanocapsule suspensions. J. Chromatogr. Sci. 48:637-640.

Fuchs J, Nitschmann WN, Packer L (1990). The antipsoriatic compound anthralin influence bioenergic parameters and redox properties of energy transducing membranes. J. Investig. Dermatol. 94:71-76.

Gambhire MS, Bhalekar MR, Gambhire VM (2011). Statistical optimization of dithranol-loaded solid lipid nanoparticles using factorial design. Brazil. J. Pharm. Sci. 47:503-511.

Golant AK, Guttman-Yassky BSE (2011). Psoriasis Treatments: A review of the current research pipeline. Psoriasis Forum 17:11-23.

Greaves MW, Hensby CN, Plummer NA, Warin AP (1978). The effect of combined ultraviolet $A$ irradiation and oral psoralens (PUVA) on skin arachidonic acid and prostaglandin concentration in psoriasis. Br. J. Pharmacol. 63:372P

Heidenreich R, Röcken MR and Ghoreschi K (2009). Angiogenesis drives psoriasis pathogenesis: Int. J. Exp. Pathol. 90:232-248.

Hern S, Allen MH, Sousa AR, Harland CC, Barker JN, Levick JR, Mortimer PS (2001). Immunohistochemical evaluation of psoriatic plaques following selective photothermolysis of the superficial capillaries. Br. J. Dermatol. 145:45-53.

Heusehkel S, Goebel A, Neubert RH (2008). Microemulsion-modern colloidal carrier for dermal and transdermal drug delivery. J. Pharm. Sci. 97:603-631.

Heydendael VM, Spuls PI, Opmeer BC, de Borgie CA, Reitsma JB, Goldschmidt WF, Bossuyt PM, Bos JD, de Rie MA (2003). Methotrexate versus cyclosporine in moderate-to-severe chronic plaque psoriasis. N. Engl. J. Med. 349:658-665.

Holick MF, Chimeh FN, Ray S (2003). Topical PTH (1-34) is a novel, safe and effective treatment for psoriasis: A randomized selfcontrolled trial and an open trial. Br. J. Dermatol. 149:370-376.

Ibbotson SH (2002). Topical 5-aminolaevulinic acid photodynamic therapy for the treatment of skin conditions other than non-melanoma skin cancer. Br. J. Dermatol. 146:178-188.

Jain S, Mittal A, Jain AK (2011). Enhanced topical delivery of cyclosporine-A using PLGA nanoparticle as carrier. Curr. Nanosci. 7:524-530.

Karasulu HY (2008). Microemulsions as novel drug carriers: the formation, stability, applications and toxicity. Expert Opin. Drug Deliv. 5:119-135.

Katare OP, Raza K, Singh B, Dogra S (2010). Novel drug delivery systems in topical treatment of psoriasis: Rigors and vigors. Indian J. Dermatol. Venereol. Leprol. 76:612-621.

Khandavilli S, Panchagnula R (2007). Nanoemulsions as versatile formulations for paclitaxel delivery: Peroral and dermal delivery studies in rats. J. Investig. Dermatol. 127:154-162.

Khokhra S, Diwan A (2011). Microemulsion based transdermal drug delivery of tea tree oil. Int. J. Drug Dev. Res. 3:191-198.

Knudsen NO, Ronholt S, Salte RD, Jorgensen L, Thormann T, Basse LH, Hansen J, Frokjaer S, Foqed C (2012). Calcipotriol delivery into the skin with PEGylated liposomes. Eur. J. Pharm. Biopharm. 81:532539.

Kreilgaard M (2002). Influence of microemulsions on cutaneous drug delivery. Adv. Drug Del. Rev. 54: S77-S98.

Kumar V, Parmar NS (2003). Herbs: A potential source for the development of new pytomedicinals. Pharma. Rev. 1:59-63.

Lakshmi PK, Devi GS, Bhaskaran S, Sacchidanand S (2007). Niosomal methotrexate gel in the treatment of localized psoriasis: Phase I and phase II studies. Indian J. Dermatol. Venereol. Leprol. 73:157-161.

Lebwohl M, Ting P Koo J (2005). Psoriasis treatment: Traditional therapy. Ann. Rheum. Dis. 64:ii83-ii86.

Leon A, Nguyen A, Letsinger J, Koo J (2007). An attempt to formulate an evidence-based strategy in the management of moderate-tosevere psoriasis: A review of the efficacy and safety of biologics and prebiologic options. Expert Opin. Pharmacother. 8:617-632.

Li J, Li X, Zhang Y, Zhou XK, Yang HS, Chen XC, Wang YS, Wei YQ, Chen LJ, Hu HZ, Liu CY (2010). Gene therapy for psoriasis in the K14-VEGF transgenic mouse model by topical transdermal delivery of interleukin-4 using ultradeformable cationic liposome. J. Gene Med. 12:481-490.

Lin YK, Huang ZR, Zhuo RZ, Fang JY (2010). Combination of calcipotriol and methotrexate in nanostructured lipid carriers for topical delivery. Int. J. Nanomed. 5:117-128.

Lowes MA, Bowcock AM, Krueger JG (2007). Pathogenesis and therapy of psoriasis. Nature 445:866-873.

Lowes MA, Lew W, Krueger JG (2004). Current concepts in the immunopathogenesis of psoriasis. Dermatol. Clin. 22:349-369.

MacDonald A, Burden AD (2007). Psoriasis: Advances in pathophysiology and management. Postgrad. Med. J. 83:690-697.

Mahrle G, Bonnekoh B, Ghyczy M, Wiegrebe W (1991). Stability of anthralin in liposomal phospholipids. Arch. Dermatol. Res. 283:483484.

Marianecci C, Rinaldi F, Mastriota M., Pieretti S, Trapasso E, Paolino D, Carafa M (2012) Anti-inflammatory activity of novel ammonium glycyrrhizinate/niosomes delivery system: Human and murine models. J. Control Release 164:17-25.

Mehnert W, Mader K (2012). Solid lipid nanoparticles: Production, characterization and applications. Adv. Drug Deliv. Rev. 64(Suppl):83-101.

Meidan V, Alhaique F, Touitou E (1998). Vesicular carriers for topical delivery. Acta Technol. Legis Med. 9:1-6.

Moghimi SM, Patel HM (1993). Current progress and future prospects of liposomes in dermal drug delivery. J. Microencapsul. 10:155-162.

Morganti P, Ruocco E, Wolf R, Ruocco V (2001). Percutaneous absorption and delivery systems. Clin. Dermatol. 19:489-501.

Mulik R, Mahadik K, Paradkar A (2009). Development of curcuminoids loaded poly(butyl) cynoacrylate nanoparticles: Physiochemical and characterization and stability study. Eur. J. Pharm. Sci. 37:395-404.

Muller RH, Petersen RD, Hommoss A, Pardeike J (2007). Nanostructured lipid carriers (NLC) in cosmetic dermal products. Adv. Drug Deliv. Rev. 59:522-530.

Nagle A, Goyal AK, Kesarla R, Murthy RSR (2011) Efficacy study of vesicular gel containing methotrexate and menthol combination on parakeratotic rat skin model. J Liposome Res. 21:134-140.

Nam SH, Ji XY, Park JS (2011). Investigation of tacrolimus loaded nanostructured lipid carriers for topical drug delivery. Bull. Korean Chem. Soc. 201:956-960.

Namdeo A, Jain NK (1996). Niosomes as Drug Carriers. Indian J. Pharm. Sci. 58:41-46.

Naldi L, Gambini D (2007). The clinical spectrum of psoriasis. Clin. Dermatol. 25:510-518.

Nanjwade B, Patel JM (2011). Liposome and proliposome based drug delivery: Paclitaxel loaded proliposome. LAP LAMBERT Acad. Pub. pp. 4-5.

Nickoloff BJ (1999). The immunologic and genetic basis of psoriasis. Arch. Dermatol. 135:1104-1110.

Patel SS, Patel MS, Salampure B, Vishwanath B, Patel NM (2010). Development and evaluation of liposomes for topical delivery of Tacrolimus (Fk-506). J. Sci. Res. 2(3):587-598.

Peeters P, Ortonne JP, Sitbon R, Guignard E (2005). Costeffectiveness of once-daily treatment with calcipotriol/betamethasone dipropionate followed by calcipotriol alone compared with tacalcitol in the treatment of psoriasis vulgaris. Dermatology 211:139-145.

Peltola S, Saarinen-Savolainen P, Kiesvaara J, Suhonen TM, Urtti A (2003) Microemulsions for topical delivery of estradiol. Int. J. Pharm. 254:99-107.

Pople PV, KK Singh (2011). Development and evaluation of colloidal modified nanolipid carrier: Application to topical delivery of Tacrolimus. Eur. J. Pharm. Biopharm. 79:82-94.

Prohić A, Helppikangas $H$, Muhović J (2007). Tagetting psoriasis with new therapies. Medicinski Glasnik. 4:56-62.

Puglia C, Rizza L, Drechsler M, Bonina F (2010). Nanoemulsion as vehicles for topical administration of glycyrrhetic acid: Characterization and in vitro and in vivo evaluation. Drug Deliv. 17:123-129.

Raza K, Katare OP, Setia A, Bhatia A, Singh B (2012). Improved therapeutic performance of dithranol against psoriasis employing systematically optimized nanoemulsomes. J. Microencapsul. (doi: 10.3109/02652048.2012.717115). 
Raza K, Negi P, Taykar S, Shukla A, Amarji B, Katare OP (2011) Novel dithranol phospholipid microemulsion for topical application: Development, chararcterization and percutaneous absorption studies. J. Microencap. 28:190-199.

Rossi M, Pellegrino M (2009). Acitretin-associated erectile dysfunction: A case report. Cases J. 2:210.

Saraswat A, Agrawal R, Katare OP, Kaur I, Kumar B (2007). A randomized, double-blind, vehicle-controlled study of a novel liposomal dithranol formulation in psoriasis. J. Dermatol. Treat. 18:4045.

Scheinfeld JDN (2004). The use of topical tacrolimus and pimecrolimus to treat psoriasis: A review. Dermatol. Online J. 10:3.

Schmid MH, Korting HC (1996). Therapeutic progress with topical liposome drugs for skin disease. Adv. Drug Deliv. Rev. 18:335-342.

Singh A, Malviya R, Sharma PK (2011). Novasome - A breakthrough in pharmaceutical technology. Adv. Biol. Res. 5:184-189.

Singh HP, Utreja P, Tiwary AK, Jain S (2009). Elastic liposomal formulation for sustained delivery of colchicine: in vitro characterization and in vivo evaluation of anti-gout activity. AAPS J. 11:54-64.

Sintov C, Botner S (2006) Transdermal drug delivery using microemulsion and aqueous systems: Influence of skin storage conditions on the in vitro permeability of diclofenac from aqueous vehicle systems. Int. J. Pharm. 311:55-62.

Solans C, Izquierdo P, Nolla J, Azemar N, García-Celma MJ (2005). Nano-emulsions. Curr. Opin. Coll. Interf. Sci. 10:102-110.

Srisuk P, Thongnopnua P, Raktanonchai U, Kanokpanont S (2012) Physico-chemical characteristics of methotrexate-entrapped oleic acid-containing deformable liposomes for in vitro transepidermal delivery targeting psoriasis treatment. Int. J. Pharm. 427:426-434.

Takahashi H, Ibe M, Kinouchi M, Ishida-Yamamoto A, Hashimoto Y, lizuka $H$ (2003). Similarly potent action of 1, 25-dihydroxyvitamin D3 and its analogues, tacalcitol, calcipotriol, and maxacalcitol on normal human keratinocyte proliferation and differentiation. J. Dermatol. Sci. 31:21-28.

Touitou E (2002). Drug delivery across the skin. Exp. Opin. Biol. Ther. 2:723-733.

Trapasso E, Cosco D, Celia C, Fresta M, Paolino D (2009). Retinoids: New use by innovative drug-delivery systems. Expert Opin. Drug Deliv. 6:465-483.

Trotta M, Peira E, Carlotti ME, Gallarate M (2004). Deformable liposomes for dermal administration of methotrexate. Int. J. Pharm. 270:119-125.
Uchegbu IF, Florence AT (1995). Non-ionic surfactant vesicles (niosomes) physical and pharmaceutical chemistry. Adv. Coll. Interf. Sci. 58:1-55.

Uchegbu IF, Vyas SP (1998). Non-ionic surfactant based vesicles (niosomes) in drug delivery. Int. J. Pharm. 172:33-70.

Umezawa Y, Ozawa A (2007). Optimal time for therapeutic drug monitoring of cyclosporine microemulsion in patients with psoriasis. Int. J. Dermatol. 46:763-766.

Urbàn-Morlàn Z, Ganem-Rondero A, Megloza-Contreas LM, EscobarChàvez JJ, Nava-Arzaluz MG, Quintanar-Guerrero D (2010). Preparation and characterization of solid lipid nanoparticles containing cyclosporine by the emulsification-diffusion method. Int. J. Nanomedcine. 5:611-620.

Walter JF, Stoughton RB, DeQuoy PR (1978). Suppression of epidermal DNA synthesis, suppression by ultraviolet light, coal tar and anthralin. Br. J. Dermatol. 99:89-96.

Weinstein GD, Koo JY, Krueger GG, Lebwohl MG, Lowe NJ, Menter MA, Lew-Kaya DA, Sefton J, Gibson JR, Walker PS (2003). Tazarotene cream in the treatment of psoriasis: Two multicenter, double-blind, randomized, vehicle-controlled studies of the safety and efficacy of tazarotene creams $0.05 \%$ and $0.1 \%$ applied once daily for 12 weeks. J. Am. Acad. Dermatol. 48:760-767.

Weyenberg W, Filev P, Van den Plas D, Vandervoort J, De Smet K, Sollie P, Ludwig A (2007). Cytotoxicity of submicron emulsions and solid lipid nanoparticles for dermal application. Int. J. Pharm. 337:291-298.

Witman PM (2001). Topical therapies for localized psoriasis. Mayo Clin. Proc. 76:943-949.

Xia YP, Li B, Hylton D, Detmar M, Yancopoulos GD, Rudge JS (2003). Transgenic delivery of VEGF to mouse skin leads to an inflammatory condition resembling human psoriasis. Blood 102:161-168.

Zhang LW, Al-Suwayeh SA, Hung CF, Chen CC, Fang JY (2011). Oil components modulate the skin delivery of 5 -aminolevulinic acid and its ester prodrug from oil-in-water and water-in-oil nanoemulsions. Int J. Nanomed. 6:693-704

Zhang Y, Gao J, Zheng H, Zhang R, Han $Y$ (2011). The preparation of 3, 5-dihydroxy-4-isopropylstilbene nanoemulsion and in vitro release. Int. J. Nanomed. 6:649-657.

Zhi XH, Jin L, Yang CG (2011). Preparation and quality evaluation of methotrexate microemulsion. China Pharm. 05:433-436. 